Georgetown University Institutional Repository http://www.library.georgetown.edu/digitalgeorgetown

The author made this article openly available online. Please tell us how this access affects you. Your story matters.

Carlson, K. A., \& Bond, S. D. (2006). Improving Preference Assessment: Limiting the Effect of Context Through Pre-exposure to Attribute Levels. Management Science, 52(3), 410-421.

doi: http://dx.doi.org/10.1287/mnsc. 1050.0434

Collection Permanent Link: hdl.handle.net/10822/1

(C) 2006 The Institute for Operations Research and the Management Sciences (INFORMS).

This material is made available online with the permission of the author, and in accordance with publisher policies. No further reproduction or distribution of this copy is permitted by electronic transmission or any other means. 


\title{
Improving Preference Assessment: Limiting the Effect of Context Through Pre-exposure to Attribute Levels
}

\author{
Kurt A. Carlson, Samuel D. Bond \\ Fuqua School of Business, Duke University, Durham, North Carolina 27708 \\ \{kurt.carlson@duke.edu, samuel.bond@duke.edu\}
}

\begin{abstract}
$\mathrm{T}$ his paper introduces a technique for improving preference assessment by reducing the influence of context on preferential choices. We propose that a decision maker who is exposed to relevant attribute levels will form spontaneous valuations, which will then insulate the decision maker from the effects of context during subsequent preference assessment. Results from three studies supported this hypothesis. Pre-exposure to product attribute levels undermined the impact of attribute priming, decision framing, and asymmetric dominance on preferential choices. A fourth study demonstrated that similar results can be obtained by allowing decision makers to pregenerate lists of attribute levels on their own.
\end{abstract}

Key words: preference assessment; context; biases; exposure to attribute levels

History: Accepted by Detlof von Winterfeldt, decision analysis; received July 12, 2004. This paper was with the authors 3 months for 1 revision.

\section{Introduction}

Preference assessment has received substantial attention from researchers interested in managerial judgment and decision making (e.g., Delquie 1993). Unfortunately, measuring preferences is difficult because even seemingly trivial differences in context can dramatically alter reported preferences (e.g., Slovic et al. 1977). These "context effects" are so pervasive that researchers in decision sciences, economics, marketing, and psychology have called for better preference elicitation methods (Bettman et al. 1998, Bless et al. 2000, Gregory et al. 1993, Hammond et al. 1999, Payne et al. 1999, Plott 1996).

Heeding recent calls for prescriptive work targeting the improvement of managerial decision making (Smith and von Winterfeldt 2004), this paper introduces a method for reducing the effect of context on observed preferences. By doing so, we hope to provide scholars and practitioners with a more accurate means of preference assessment. We propose that prechoice exposure to a list of attribute levels (e.g., the bottom of a backpack may be made of leather, nylon, or rubber) yields spontaneous valuations (e.g., "I like nylon," "I don't like leather"), which insulate decision makers against context during subsequent choices. In this way, preferences measured after exposure to attribute levels should more accurately reflect true preferences than those measured without such pre-exposure. This idea is developed and tested in a series of preferential choice studies.

\section{Context Effects}

Although some scholars define context narrowly as the set of alternatives under consideration, we use the term to represent any irrelevant information that is made temporarily available by the choice setting (Bless et al. 2000, Higgins 1996), and, therefore, is immaterial to the experienced utility resulting from a choice. It is important to distinguish between the environment in which a decision is made (e.g., the temperature outside) and the irrelevant choice context. Satisfaction with a chosen option should and often does depend on the environment (e.g., preference for a cold drink on a hot day and a hot drink on a cold day), but it should not depend on irrelevant context (e.g., display characteristics, framing effects, set composition, etc.). Nevertheless, such dependencies are frequently observed, and these context effects have received considerable attention as a source of bias in utility assessment (e.g., von Winterfeldt and Edwards 1986). Spanning a range of disciplines, researchers have found variation in reported preferences due to changes in task instructions (Dhar et al. 1999, Shafir 1993), preference elicitation method (Hsee 1996, Tversky et al. 1988, Lichtenstein and Slovic 1971), visual background (Mandel and Johnson 2002), social environment (Ariely and Levav 2000, Ratner and Kahn 2002), choice set composition (Huber et al. 1982, Nowlis et al. 2002, Simonson and Tversky 1992, Simonson 1989), information framing (Janiszewski 
et al. 2003, Janiszewski and Cunha 2004), and information order (Bond et al. 2006, Carlson et al. 2006, Tyszka and Wielochowski 1991). All these examples violate the normative principle of preference invariance, which states that preference towards particular objects should depend only on features of the objects themselves (Tversky and Kahneman 1986). Applying this principle to utility assessment, Schoemaker and Hershey (1992) suggest that discrepancies among seemingly equivalent methods (e.g., judgments of certainty equivalence versus probability equivalence) have multiple underlying causes.

The combined effects of environment and context can be understood by considering the different elements contributing to observed preference. For example, when a measure of product preference is obtained for a particular individual within a particular setting, the outcome will be a function of: (1) true/latent preference for that product in the current environment, (2) effects of irrelevant contextual variables, and (3) random noise. Under this formulation, systematic changes in observed preference may result either from bias induced by the temporarily prevailing context or from changes in the general environment that cause the underlying preference itself to change. ${ }^{1}$ Holding the environment constant, therefore, a criterion by which to evaluate alternative assessment methods is their ability to yield the same observed preferences despite changes in irrelevant context that normally induce different preferences. In other words, a method that mitigates context effects on choice is better than one that does not, because such a method will, on average, produce less biased estimates of underlying preference.

Most existing procedures for improving preference assessment advise careful consideration of the alternatives (e.g., Baron 1997, Hammond et al. 1999). Frequently, these approaches call for remedies that are specific to the particular context effect of concern (e.g., Payne et al. 1999, Russo and Carlson 2002). For example, to avoid scale compatibility effects, Delquie (1997) proposes a bidimensional matching procedure whereby respondents adjust attribute levels for both alternatives simultaneously. To mitigate response scale effects, Huber et al. (1993) advise questioners to solicit responses in multiple ways using different response scales. Similarly, the consideration of multiple alternatives and problem representations has been advocated as a means of reducing framing effects (Hammond et al. 1999, Russo and Schoemaker 2002). More general debiasing procedures exist (e.g., Gregory et al. 1993, Keeney 1996), but these involve

\footnotetext{
${ }^{1}$ For present purposes, we ignore any systematic errors that result from an individual's use of different strategies at different times (Delquie 2003).
}

substantial complexity, making them too arduous for most choices worthy of thought. The need exists for a simpler means by which the influence of context on preference assessment can be overcome.

\section{Spontaneous Valuation of Attribute Levels}

Recent evidence suggests that individuals often form automatic positive or negative evaluations upon exposure to a stimulus (Bargh 1996, Jarvis and Petty 1996, Musch and Klauer 2003). For example, researchers have obtained physiological and scaleresponse data indicating that individuals spontaneously evaluate human faces (Livingston and Brewer 2002), abstract art (Duckworth et al. 2002), and even nonsense words (Garcia and Bargh 2003). Importantly, spontaneous valuation occurs even when respondents are not explicitly instructed to form affective reactions (Duckworth et al. 2002, Zajonc 1980).

The method we propose utilizes spontaneous valuation of relevant attribute levels prior to preference assessment. To illustrate, consider the following excerpt from Consumerreports.com that describes an attribute category (multichannel surround sound) for DVD players:

Multichannel Surround Sound. Dolby Digital decoding built-in refers to circuitry that lets a DVD player decode the six-channel audio encoded into DVD discs; without the built-in circuitry, you'd need to have the decoder built into the receiver or use a separate decoder box to take advantage of six-channel audio. Some DVD players also may support Digital Theater System (DTS) decoding for titles using the six-channel encoding format. When you're watching DVD-based movies, dynamic audio-range control helps keep explosions and other noisy sound effects from seeming too loud.

In addition to describing the components that underlie the multichannel surround sound attribute category, this description introduces various levels for each component. The components in this example take on one of two discrete levels (e.g., a DVD player can offer dynamic audio range control or not). However, components in other attribute categories can have multiple levels (e.g., the brand or color of the DVD player) and they can be continuous (e.g., watts per channel or price). We expect that exposure to attribute levels before a choice will yield spontaneous valuations (e.g., "I like dynamic range audio control."). Because these valuations are formed prior to the choice process, they should be unbiased with respect to the prevailing choice context. If individuals use these (pre)valuations to construct preferences over specific options during the choice process (Einhorn 1972, Keeney 1992, Kleinmuntz 1990, Lynch 
1985, Montgomery and Willen 1999, Svenson 1996), the effect of context on observed preferences should be attenuated. ${ }^{2}$

As a debiasing technique, pre-exposure to attribute levels is related to decision analysis methods that require individuals to contemplate their values before making a choice (Gregory et al. 1993, Keeney 1992, Payne et al. 1999, Wright and Kriewall 1980). For example, Wright and Kriewall (1980) predicted the colleges to which high school seniors would apply from survey responses taken a year in advance. Before the survey, a group of forethought participants was given a list of college features and sample profiles, asked to contemplate their preferences, and given a practice choice. Utility functions derived from the survey responses of these participants were more predictive of actual college applications than were utility functions of control participants.

In contrast to such involved procedures, preexposure to attribute levels neither imparts information about specific alternatives nor requires explicit evaluation in advance of the choice process. Both of these aspects are important advantages of the procedure, because it has been shown that simply thinking about an attitude object (a product, course of action, etc.) can lead to polarization of one's attitude toward that object (Chaiken and Yates 1985). For example, in a choice between two options, even minimal information about the alternatives can cause decision makers to establish a tentative preference, toward which new information is distorted (Carlson and Russo 2001, Russo et al. 2000). The pre-exposure technique prevents attachment to a particular option, because no information is revealed about specific items within the choice set. The simplicity of the technique is therefore a methodological advantage as well as a practical one.

Despite its simplicity compared to other procedures, there is reason to believe that attribute level pre-exposure may reduce the impact of context on reported preferences. Recent work by Carlson and Pearo (2004) examined the tendency to predecisionally distort new information in favor of a tentatively preferred (leading) option. During the choice process, participants who had examined attribute levels in advance did not distort attribute evaluations to support their leading option, while those who had not been pre-exposed to attribute levels did exhibit distortion. Moreover, participants spent less time evaluating attributes that were described by levels they had

\footnotetext{
${ }^{2}$ The constructed preference view holds that preferences are often constructed at the time of elicitation (Payne 1982, Payne et al. 1992, Slovic 1995). Related views suggest that thought and experience help consumers uncover (Gregory et al. 1993) or discover (Plott 1996) preferences. Although differing in philosophy as to the ontogenesis of preferences, these views share the notion that preferences emerge during consideration of the choice options.
}

seen previously, suggesting that pre-exposure resulted in spontaneous valuation. Although predecisional distortion is not typically considered a context effect, the leading option may be seen as an "internal frame," specific to each individual and each attribute, that influences the preference construction process. Given the success of pre-exposure in eliminating the effect of this internal frame, we might also expect the technique to mitigate the influence of irrelevant cues in the external decision environment-that is, to undermine the effects of context.

Our research hypothesis is as follows: Choices made by individuals who are pre-exposed to attribute levels will be less influenced by context than those made by individuals not pre-exposed to attribute levels. We test this hypothesis in four preferential choice studies using three diverse context effects: (1) attribute priming (Mandell and Johnson 2002, Bettman and Sujan 1987), (2) decision framing (Ganzach 1995, Shafir 1993), and (3) asymmetric dominance (Huber et al. 1982, Simonson 1989). In Studies 1-3, we cross pre-exposure to a list of attribute levels (exposure versus no-exposure) with two levels of prevailing context. Data to support the hypothesis would take the form of a choice difference across contexts in the no-exposure condition but not the pre-exposure condition. In Study 4, we alter the exposure technique by allowing participants to generate attribute levels without external aid. Participants in all four studies are undergraduate and MBA students. To demonstrate the applicability of the pre-exposure procedure to ordinary decisions, stimuli for the studies are products and services familiar to this population. However, just as context effects are robust across a variety of domains, we expect that our results will extend to other choice settings.

\section{Study 1: Attribute Priming}

\section{Overview}

Cognitive psychologists have compellingly demonstrated the ability of supraliminal and subliminal primes-stimuli presented with little or no awareness by the individual-to affect thought and behavior (e.g., Greenwald et al. 1996). Extending this idea to consumer preference formation, Mandel and Johnson (2002) investigated the effects of attribute primes on information search and choice. Participants in one study were given a choice between two products (e.g., couches) that were described on four attributes (price, comfort, styling, and dimensions). One of the couches was more comfortable and the other was less expensive. The background display was varied to show either pennies or clouds, creating supraliminal primes of either price or comfort, respectively. As expected, participants preferred the couch that was superior 
on the primed attribute. Mandel and Johnson (2002) attributed their result to a shift in attribute focus that influenced the preference construction process. If preference construction is indeed at the heart of this effect, then pre-exposure to attribute levels may undermine the ability of a prime to influence product preferences.

\section{Methods}

Participants and Design. Participants were 68 undergraduate students. All participants reported owning a bag (often a backpack) that they used to carry books. Participants received extra course credit for their participation. Each participant was assigned to one of four conditions derived by crossing attribute prime (price versus comfort) with pre-exposure to an attribute-level list (no versus yes).

Materials and Procedure. We created a conceptual replication of Mandel and Johnson's (2002) choice, using two backpacks described by four attributes (cleaning, dimensions, wearability, and price). Each attribute listed various attribute levels for both backpacks. For example, the wearability attribute read as follows:

Backpack $\mathrm{J}$ is made of thick weave nylon. The twoway adjustable shoulder straps are padded and flared to better disperse the weight of the pack. All stitching is opposite points of contact with the body to avoid chaffing. The average thickness of the foam padding is $1 \frac{1}{2}$ inches.

Backpack $\mathbf{T}$ is made of rugged canvas packcloth. All seams are double stitched for durability. The shoulder and sternum straps can be adjusted to fit almost any adult. On average, the bag's foam padding measures 1 inch thick.

The price and wearability attributes were pretested so that Backpack J was more comfortable and Backpack $T$ was less expensive. The other two attributes presented information that was pretested to be neutrally valenced, yet ambiguous enough to be seen as favoring either backpack by any particular person.

Using clipart images of either clouds or dollar bills on the upper corners of the page, participants were supraliminally primed with price or comfort. Following Mandel and Johnson (2002), we expected that these primes would lead individuals to construct product-level preferences that favored either the cheaper or more wearable backpack, respectively. The attribute prime factor was crossed with a second factor, pre-exposure to attribute levels. Participants in the no-exposure condition saw the four attribute category names and descriptions before encountering the product-specific information. For example, backpack wearability was described as follows:
Wearability: The construction of a backpack influences its comfort. Factors to consider include materials, stitching, straps, and padding.

In contrast, participants in the pre-exposure condition were given attribute names, definitions, and a list of attribute levels for each of the four attribute categories. For example, the wearability information for those in the pre-exposure condition added the following:

Wearability: ...Packs can be made of canvas, nylon, polyester, rubber, or some combination of these. Stitches can be hidden or exposed; exposed stitches that make contact with the body can cause chaffing. Strap adjustment helps ensure proper fit, and padding ranges from $1 / 2$ inch to $1 \frac{1}{2}$ inches thick. A thicker padding keeps the body from being jabbed by the contents of the bag.

This paragraph lists several attribute levels for the attribute category. For example, the pack can be made of "canvas, nylon, polyester, rubber, or some combination of these." (The complete stimuli used to manipulate pre-exposure for Studies 1-3 are presented in the appendix.) Importantly, the added lines in the preexposure condition reveal nothing about the actual composition of the backpacks to be presented. Thus, pre-exposure does not allow participants to infer the attribute levels that will characterize the backpacks and, therefore, does not allow them to form relative preferences in advance. Instead, we expected that participants exposed to this information would form attribute-level valuations (e.g., "I don't like nylon.") and that these valuations would be available during the later choice process, thereby undermining the prime's ability to influence backpack preferences.

Each participant was given a choice packet containing the study materials and was instructed to proceed at their own pace. On average, the task took approximately five minutes. The first page of the packet contained the cover story and the preexposure manipulation (described above). The second page listed the wearability and price attributes (counterbalanced), with the background prime repeated. The third and final page presented the remaining two attributes and asked participants to make their selection.

\section{Results}

We computed the proportion of participants that preferred the cheaper backpack for all four conditions. Participants in the no-exposure condition were more likely to prefer the cheaper backpack when primed with price $(46.7 \%)$ than when primed with comfort $(11.1 \% ; z=2.14, p<0.05)$. Thus, the attribute priming effect was reproduced for participants in the no-exposure condition. As expected, pre-exposure participants did not prefer the cheaper backpack more 
when primed with price $(7.1 \%)$ than when primed with comfort $(28.6 \% ; z=1.54, p>0.50)$, suggesting that the attribute prime was unsuccessful for these participants.

\section{Discussion}

Study 1 replicated Mandel and Johnson's (2002) attribute priming effect on product choice, but only for participants who were not exposed to attribute levels before the choice process. Although participants in the pre-exposure condition saw the kind of information that would later be used to describe the backpacks, they did not know which specific attribute levels would describe which backpack. Moreover, many of the attribute levels seen during pre-exposure (e.g., polyester or rubber materials) did not describe either of the backpacks presented. Therefore, it was not possible for participants to construct preferences for specific backpacks during pre-exposure. Instead, the data support our claim that pre-exposed participants based their preferences on previously formed valuations of the attribute levels to which they had been exposed. In other words, without the ability to bias attribute-level valuations, the attribute prime did not influence product-level preferences. The next study extends the research hypothesis to a different context effect, decision framing.

\section{Study 2: Selection vs. Rejection}

\section{Overview}

While Study 1 manipulated context by the insertion of attribute primes, other context effects are more closely linked to the decision task itself. One line of research has focused on preference reversals that result from instructions to "select" the options one prefers or "reject" the options one does not prefer (Shafir 1993, Ganzach 1995). The effect of task instrucutions is usually found to depend on the amount of attribute variability within the options. For example, Ganzach (1995) found that instructions to select led to increased preference for impoverished options (whose attribute levels are moderate), while instructions to reject led to increased preference for enriched options (whose attribute levels are more extreme). In contrast, Shafir (1993) found that impoverished options were relatively more preferred under instructions to reject than instructions to select. Wedell (1997) reconciled these two findings by observing that the impoverished option was more attractive in Ganzach's studies (i.e., it was preferred by $75 \%$ of participants), while the reverse was true in Shafir's studies (only $44 \%$ preferred the impoverished option). ${ }^{3}$ Because all of

\footnotetext{
${ }^{3}$ Wedell's (1997) account holds that increased commitment under selection instructions guides individuals to prefer the option that is slightly more attractive on average.
}

these authors implicate preference construction as the process underlying the instruction-framing effect (see also Meloy and Russo 2004), we expected that preexposure to attribute levels would reduce the impact of task instructions on reported preference.

\section{Methods}

Participants and Design. Participants were 204 undergraduate students at a large university who were compensated $\$ 5$ for participation in this and other unrelated studies. Each participant was assigned to one of four conditions created by crossing pre-exposure (no versus yes) with choice frame (accept versus reject).

Materials and Procedure. Participants were told to imagine that they were planning a beach vacation for which they had narrowed their options to two destinations. Having made reservations at both destinations, they now had to decide on one. Before reading about the options, the pre-exposure manipulation was administered. As in Study 1, participants in the no-exposure condition were given simple descriptions for each of five categories (beaches, hotel, nightlife, water temperature, and weather). For example, noexposure participants viewed the following information regarding nightlife:

Nightlife: This indicates the amount of nightlife in the nearby area.

Meanwhile, those in the pre-exposure condition were provided with identical names and definitions, as well as a list of levels for each attribute category. For example, the following was added to the Nightlife attribute:

Nightlife: Some vacation areas have virtually no clubs, restaurants, etc. in the proximity. Other areas have a bit more nightlife, and some offer numerous possibilities.

Complete attribute category descriptions for both conditions are provided in the appendix. After reading these descriptions, participants examined the two destination profiles and made their choice. The enriched destination was described by levels that were very favorable (e.g., gorgeous beaches) and very unfavorable (e.g., very cold water), while the impoverished option was described by levels that were moderately favorable (e.g., average beaches and mediumtemperature water). In keeping with Ganzach (1995), the balance of attribute level information slightly favored the impoverished option. The framing manipulation was embedded in the instructions: participants in the accept condition were told to circle the location for which they would keep their reservation, while those in the reject condition were told to circle the location for which they would cancel their reservation. 


\section{Results}

To compare preferences across task instructions, the preferred destination was defined as the option circled in the "accept" frame and the option not circled in the "reject" frame. Recall that Ganzach's (1995) result obtains when the impoverished option is more attractive (on average) than the enriched option. The data suggest that this precondition existed, as $57.4 \%$ of participants preferred the impoverished option, a value reliably larger than chance $(z=2.10, p<0.05)$. Next, we examined the data relevant to our preexposure hypothesis.

As in Study 1, we compared choice proportions for each decision frame across the pre-exposure and no-exposure conditions. Results were strongly supportive of the hypothesis: the effect of decision frame on choice was significant for those in the no-exposure condition $(z=2.18, p<0.05)$, but not for those in the pre-exposure condition $(z=0.32, p>0.35)$. Specifically, preferences in the no-exposure condition revealed a preference difference across the two frames, with $64.2 \%$ of participants in the accept condition preferring the impoverished option, and $44.1 \%$ in the reject condition doing so. However, the effect was nearly eliminated among participants in the preexposure condition, where $63.6 \%(60.4 \%)$ of participants in the accept (reject) condition preferred the impoverished option.

\section{Discussion}

Results replicated Ganzach's (1995) finding that framing of task instructions alters preference for an impoverished option over an enriched option; however, as predicted, the effect was reduced by exposing participants to a list of attribute levels before the choice process. These data support our contention that attribute-level preferences were formed in advance by pre-exposure, thereby insulating participants who received this exposure from the influence of decision frame. The next two studies extend the investigation to Huber et al.'s (1982) asymmetric dominance effect.

\section{Study 3: Asymmetric Dominance}

\section{Overview}

One of the most enduring context effects of the last two decades is the asymmetric dominance effect (Huber et al. 1982). This phenomenon is characterized by a change in relative preference between two options due to the inclusion of a third option that is dominated by one of them. Specifically, the dominating option is selected more often when the choice set includes an option that it dominates. Because explanations of asymmetric dominance tend to emphasize its influence on the preference construction process (e.g., Ariely and Wallsten 1995), we believe that our pre-exposure technique might undermine this effect.

\section{Methods}

Participants and Design. Participants were 108 undergraduates at a large university who were compensated $\$ 5$ for participation in this and other unrelated studies. Participants were randomly assigned to one of four conditions created by crossing preexposure to relevant attribute levels (no versus yes) with choice set composition (dominated option present versus absent).

Materials and Procedure. Stimuli examining the dominance effect have traditionally been concise, utilizing a matrix of numeric values for each option on each attribute. While the matrix format facilitates interalternative comparisons, the nonverbal aspect of this approach does not align well with our pre-exposure intervention. Therefore, short narrative attributes were constructed to describe the choice alternatives (hotels) on three attribute categories: location, pool, and services. Participants were asked to imagine that they would be spending spring break at the beach and needed to select a hotel for the vacation. All participants viewed an information matrix describing the available options, with the hotels in rows and attribute categories in columns. The choice set was varied such that participants in the 2-hotel condition saw information on only two hotels (A and B), neither of which was clearly superior. Participants in the 3-hotel condition saw information on the same two hotels and a third hotel $(C)$, which was dominated by Hotel B (but not by Hotel A). For example, the pool attribute read as follows:

Hotel A has a large outdoor swimming pool with a swim-up bar, as well as a small indoor pool with a snack shop. The hotel doesn't have a Jacuzzi, but it does offer men's and women's saunas.

Hotel B has a huge outdoor pool, with a swim-up bar and an outdoor grill. Though there is no indoor pool, two Jacuzzis are located alongside the outdoor pool.

Hotel $\mathbf{C}$ has a moderate-sized outdoor swimming pool and an outdoor grill. There is one Jacuzzi provided, but no indoor pool.

Before seeing the attribute matrix, participants encountered the pre-exposure manipulation. As in Studies 1 and 2, participants in the no-exposure condition read short descriptions of the three attribute categories. For example, the pool category description for those in the no-exposure condition was as follows:

Pool: This refers to characteristics of the pool area operated and maintained by the hotel.

As before, those in the pre-exposure condition read the same attribute descriptions, along with a list of attribute levels for each category. For the pool example, pre-exposure information included the following: 
Pool: ... Pools can vary across a range of sizes from small to enormous, and they may or may not have Jacuzzis or a sauna nearby. Hotel pools sometimes offer amenities such as swim-up bars or an outdoor grill/eatery. Some hotels also have an indoor pool.

All participants then rated the importance of the attributes by dividing 100 points among them (this measure was used to determine whether the preexposure process influenced the perceived importance of attribute categories). Participants then examined the attribute matrix information and made their hotel selection.

\section{Results}

Three participants were excluded for preferring the dominated hotel, leaving 105 participants for the analysis. The difference in the dominance effect across exposure conditions was examined by comparing choice proportions for the dominating option. As expected, preferences for those in the no-exposure condition depended on whether the dominated option was present in the choice set. That is, while $55.2 \%$ of participants in the 2 -hotel condition preferred the dominating option, $79.2 \%$ of participants in the 3-hotel condition did so $(z=1.93, p<0.05)$. However, in accordance with our prediction, this effect did not obtain for those in the pre-exposure condition, where the dominating hotel was chosen by roughly equal proportions $(82.1 \%$ and $75.0 \%$ in the 2-hotel condition and the 3-hotel condition, respectively).

Next, we examined whether attribute category weights were different across the two pre-exposure conditions. Mean importance weights did not differ across conditions for any of the attributes (all $p>$ 0.50 ). Thus, the stabilizing effect of attribute-level preexposure was not driven by changes in the perceived importance of the attribute categories.

\section{Discussion}

Participants who were exposed to attribute levels did not exhibit the asymmetric dominance effect, suggesting that these participants based their choices on previously formed attribute valuations. However, a weakness of the present procedure was that the self-reported attribute weights were collected before the choice task. Thus, participants in the pre-exposure conditions may have been motivated to be consistent with their reported weights, reducing the ability of context to operate through actual changes in underlying attribute importance. This possibility is examined in Study 4 . In addition, we modify the pre-exposure technique to broaden its applicability to everyday preference assessment.

\section{Study 4: Self-Generation and Asymmetric Dominance}

\section{Overview}

The results of Studies 1-3 indicate that by preexposing consumers to attribute levels, the influence of context on product-level preferences can be undermined. Despite its simplicity, a shortcoming of this technique is its reliance on externally provided attribute levels. Fortunately, for many common choices (such as those examined in the present studies), consumers are sufficiently knowledgeable to generate attribute levels from memory. If individuals can indeed recall relevant attribute levels, and if these recalled levels are spontaneously valued upon recall, it may be possible to reduce the influence of context and improve preference assessment with minimal outside intervention.

\section{Methods}

Participants and Design. Participants were 175 undergraduates and MBAs at a large university who were compensated $\$ 5$ for participation in this and other unrelated studies. Each participant was randomly assigned to one of four conditions determined by crossing self-generation (no versus yes) with choice set (dominated option present versus absent).

Materials and Procedure. The format of Study 4 was very similar to that of Study 3. After reading a cover story and undergoing the self-generation manipulation, participants selected a vacation hotel from either a 2-hotel or a 3-hotel set, with the latter containing a dominated option. However, Study 4 differed from the prior study in four ways. The most significant change was in the operationalization of the intervention technique. As before, both no-generation and self-generation participants read the same attribute descriptions (e.g., the location attribute description read: "The hotel's location refers to where it is situated relative to other attractions."). Unlike Studies 1-3, however, participants in the selfgeneration condition were not given a list of attribute levels, but instead were asked to generate a list on their own. For example, the self-generation group was given the following instructions for the location attribute: "On the lines below, list some of the characteristics of the location that might influence your decision." Beneath each attribute description were lines on which participants listed attribute levels that came to mind. In keeping with the other studies, participants were not asked to evaluate the information they generated, but we expected that they would do so spontaneously. In addition, descriptions of the hotels were altered slightly to make the dominating hotel 
a bit less attractive than that of Study 3, and the information grid was transposed so that attribute categories appeared in rows. Finally, for reasons indicated above, importance weights for the attribute categories were collected after (not before) participants made their choice.

\section{Results}

Six participants who selected the dominated hotel were excluded, leaving a sample size of 169. The proportion of participants choosing the dominating hotel was compared across each of the four cells. As predicted, analyses revealed that context influenced choice only for the no-generation conditions. Among these participants, $56.3 \%$ chose the dominating hotel when the dominated hotel was absent, while $76.4 \%$ did so when the dominated hotel was present-an asymmetric dominance effect of $20 \%$ ( $z=$ $1.92, p<0.05)$. However, among participants who pre-generated attribute levels, the effect was virtually eliminated: $58.1 \%$ chose the dominating hotel from the 2-hotel set, while $60.8 \%$ did so from the 3-hotel set $(z=0.26, p>0.40)$. As in Study 3, self-reported importance weights did not differ across conditions (all $p>0.25$ ), suggesting that the reduction of contextual influence did not operate through perceived importance of the attribute categories.

\section{Discussion}

Asking participants to generate attribute levels in advance of a choice process eliminated the asymmetric dominance effect. This finding provides initial evidence that having respondents generate attribute levels from memory before engaging in a choice process may be a simple and effective technique for reducing the effect of context on preference assessment. Although this particular approach is useful only when respondents possess adequate knowledge to generate meaningful attribute levels, such instances are common in everyday choice settings.

\section{General Discussion}

The data from Studies 1-4 are summarized in Figure 1. For each study, the figure shows the impact of a change in prevailing context on choice for participants with and without prior exposure to a list of relevant attribute levels. Data from the first three studies provide convergent evidence that preexposure to attribute levels mitigates the influence of context during a subsequent choice. Adding to this evidence, Study 4 demonstrates that the effect of context can be reduced by self-generation of attributelevel information.

Because none of the studies described the actual choice options during the pre-exposure process, it is unlikely that the intervention caused participants to

\section{Figure 1 Context Effects on Choice for All Studies}

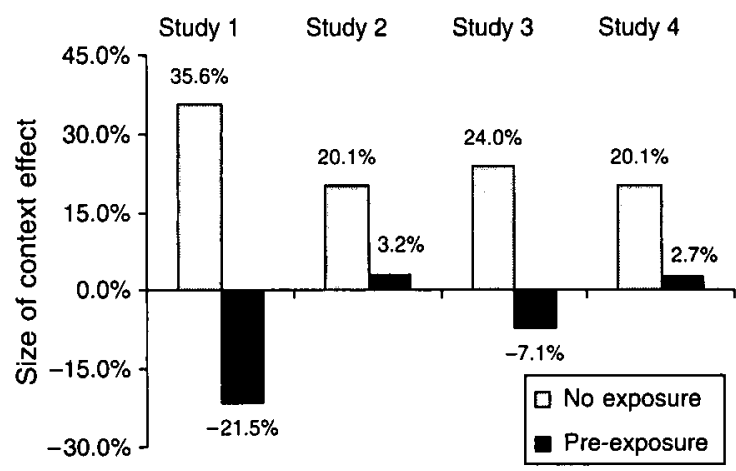

construct item-level preferences in advance. Instead, we contend that pre-exposure led participants to spontaneously value the exposed attribute levels and, later, to use these valuations as inputs during the preference construction process, thereby mitigating the effects of context. Although this explanation is consistent with other evidence that consumers spontaneously value product-related information (Carlson and Pearo 2004, Kivetz and Simonson 2000, Svenson 1996, Russo et al. 1996), the data above provide only indirect evidence that individuals in our studies spontaneously valued the attribute levels to which they were pre-exposed. To obtain more direct evidence, a follow-up study was conducted.

Using software for tracking reaction times, 89 undergraduate participants were shown six different attribute levels describing vacation destinations (similar to those used in Study 2). Participants rated the favorability of each attribute level on a nine-point scale $(1=$ extremely bad; $9=$ extremely good). The time taken to rate each attribute level was recorded, as was the time taken for a practice rating (which served as a covariate in the analysis). We reasoned that individuals who had been pre-exposed to an attribute level would require less time to provide favorability responses for that level, because valuations formed during the exposure process would still be available. To examine our hypothesis, each participant was randomly exposed to three of the six attribute levels before the evaluation task. Furthermore, we varied the instructions regarding pre-exposure: half the participants were told that they would be making a vacation choice, while the rest were told to memorize the attribute levels that were presented. The latter manipulation was included to determine whether spontaneous valuation requires knowledge of an impending choice.

To test for a facilitative effect of pre-exposure, we compared the time required by participants to rate the favorability of the three pre-exposed attributes with the time required to rate the three attributes that had not been pre-exposed (latencies were log-transformed 
to correct for strong positive skew). Results confirmed the expected pattern: evaluation times were faster for attribute levels to which participants had been preexposed ( $M=4.41$ seconds) than for those to which participants had not been pre-exposed $(M=4.55, F=$ $4.73, p<0.04)$. The effect of pre-exposure did not depend on the specific instructions provided (interaction $p>0.50$ ). In summary, this result supports our claim that individuals form spontaneous evaluative reactions as a result of the pre-exposure process. Nevertheless, other possible accounts for the effectiveness of the pre-exposure technique exist. Below we consider some of these.

\section{Alternative Accounts}

One competing explanation for our data is that the mitigation of contextual influence was caused by an increase in expertise brought about by the preexposure procedure. Such expertise might well lead to decreases in procedural variance: e.g., Coupey et al. (1998) observed fewer choice-matching preference reversals for decisions involving unfamiliar product categories than for decisions involving familiar ones. However, we find an explanation based solely on expertise unlikely, given that participants were probably already knowledgeable in the familiar domains examined here. Moreover, Sen (1998) found that experts exhibit more (not less) asymmetric dominance when alternatives are described verbally (as was the case in Studies 3 and 4).

Another alternative to our spontaneous valuation account is that pre-exposure to attribute levels increased activation of an accuracy goal (Johnson and Payne 1985), perhaps causing participants to process the information more rigorously. We see this as unlikely for two reasons. First, previous research suggests that effort tends to enhance rather than diminish the effects of prevailing context (e.g., Dhar et al. 2000). Second, unpublished data from the studies reported in Carlson and Pearo (2004) shows no effect of attribute-level pre-exposure on reported effort.

A different alternative explanation is based on perceptual changes that might have been caused by the pre-exposure process. It is commonly accepted that prior exposure to a stimulus increases the fluency with which individuals perceive it, which in turn increases overall liking for that stimulus (Zajonc 2001). Applied to the present studies, a fluency account predicts that all options would be seen as more attractive in the pre-exposure conditions; however, this account would not predict the observed changes in relative choice shares. In a similar vein, prior exposure may have created a sort of "preference fluency," manifesting itself as a feeling of greater ease with the choice. This account also seems unlikely, as increasing preference fluency (by making information easier to read; see Novemsky et al. 2003) has been found to exacerbate the asymmetric dominance effect.

A final possibility is that pre-exposure to attribute levels enhanced the salience of certain attributes at the expense of others, with the result that preferences became more dependent on attributes whose salience had increased. We offer two responses to this admittedly plausible concern. First, in Studies 3 and 4, self-reported attribute weights did not reveal any difference across exposure conditions. Second, even if pre-exposure is assumed to alter the attribute weights used in preference formation, it is not clear how such changes would reduce the impact of the context effects presented here (e.g., a dominance relationship does not depend on a particular weighting of attributes).

\section{Limitations and Future Research}

The robustness of pre-exposure is evident in its ability to mitigate all three context effects considered in these studies. However, we admit the possibility that other effects may be more resistant to the technique; application of pre-exposure to a wider variety of context phenomena would thus be a worthwhile endeavor. For example, if the process works (as we argue) by limiting biases in the construction of attribute-level valuations during the choice process, then context effects that operate solely through other mechanisms may not be impacted by pre-exposure. Another factor that may moderate the effectiveness of the method is the degree to which the attribute levels themselves are conducive to spontaneous valuation. Although researchers have considered the overt evaluability of various types of attributes (Hsee 1996), surprisingly little work has focused on differences in the conduciveness of attributes to automatic evaluation. Relevant work by Hsee and Rottenstreich (2004) finds that certain types of targets are likely to be valued by reliance on "feeling," while other types invoke a reliance on "calculation." It is possible that preexposure will be less effective for attribute levels of the latter type, which may not lend themselves to immediate evaluative response.

The present studies were concerned with reliability at the group level. This reliability is a necessary but insufficient indicator that the assessments were indicators of latent, context-free values. To make a more compelling case, the procedure should be tested at the level of the individual. For example, participants could be given a series of choices with and without prior attribute-level exposure. Similarly, a pretestposttest design could be utilized: after an initial choice made without pre-exposure (and a subsequent delay), participants could be asked to choose again, this time with the benefit of the technique. Finally, preferences obtained after pre-exposure could be examined for 
agreement with those obtained via conjoint analysis or other standard analytical tools.

On a related note, we cannot say whether preferences obtained in the pre-exposure conditions were "better" in an absolute sense. Presumably, an ideal elicitation process would yield preferences that are independent of irrelevant context and that maximize long-run satisfaction. Delquie (2003) suggests three factors that contribute to random variability in preference assessment: (1) unreliability in the elicitation protocol, (2) lack of attentiveness or motivation, and (3) inherent lack of precision in preferences. If preexposure to attribute levels decreases the withinperson idiosyncrasies in decision strategy use, it may undermine these sources of variability and limit both systematic fluctuations (as demonstrated in the studies above) and nonsystematic fluctuations in preference reports. Future research might examine whether pre-exposure to attribute levels yields preferences that are temporally stable. Such an outcome would suggest that the benefits of pre-exposure extend beyond contextual invariance to long-run decision satisfaction.

Future research should delve deeper into the process underlying the context mitigation demonstrated in these studies. Our latency data provide an initial step in this direction, but numerous other tools are available. Thought listings or verbal protocols might be gathered to provide deeper insight into the effects of the intervention on information processing. The vividness of the exposure information might be varied to determine what minimum level of richness is required. Finally, individual difference variables might be used to examine whether pre-exposure is most effective for persons inclined to react strongly to stimuli or hold strong opinions; Jarvis and Petty's (1996) "need to evaluate" is an example of one such variable.

\section{Conclusion}

This paper introduced and evaluated a technique for improving preference assessment. Evidence from four studies suggests that pre-exposure to a list of relevant attribute levels allowed individuals to spontaneously establish context-free valuations for attribute levels, thereby reducing the influence of context on preference construction during a subsequent choice process. This technique provides a simple way to enhance the likelihood that observed preference reports are representative of actual underlying preferences. Accordingly, pre-exposure offers clear value to managers wishing to better understand customer or supplier preferences. Additionally, by making irrelevant choice context less influential in consumer decision making, this technique may contribute meaningfully to consumer welfare.

\section{Acknowledgments}

The authors thank Jim Bettman, Joel Huber, Ralph Keeney, John Payne, and Jay Russo for helpful comments. This paper has benefitted from comments by Detlof von Winterfeldt (the departmental editor) and three anonymous referees.

\section{Appendix. Pre-exposure Manipulations for Studies 1-3}

Regular text was observed by all participants. Italicized text was observed only by those in the pre-exposure conditions.

\section{Study 1: Backpacks}

Cleaning: Some backpacks are less difficult to keep clean than others, either because their materials are more easily washable or because they better resist dirt, stains, etc. Sooner or later, every backpack needs cleaning. Machine washing is the easiest method, but dry cleaning is also an option-though most people don't think of it or don't want to pay for it. Most backpacks are machine washable, but some require hand washing. Shrinkage is also a concern. Some materials resist shrinkage (e.g., nylon) better than others (e.g., canvas). Finally, if machine washing will be frequent, consider how well the backpack will hold up.

Dimensions and Pockets: The size of the backpack that you need should be anticipated. It is important to consider not only the capacity, but also the dimensions of the pack. Some people don't like backpacks that are too wide or too narrow, too shallow or too deep. Pockets describe the number of pockets in and on the backpack, as well as the sizes of those pockets. Some backpacks may have many smaller pockets, and others may have fewer large ones.

Price: One of the important factors shoppers consider when considering a backpack is its cost, especially considering that a backpack can be an important long-term investment. College students tend to have limited disposable income, and since they make up the majority of backpack purchasers, it is important that prices not be excessive in order to attract buyers. The average backpack on the market costs $\$ 50.00$, but there is a wide range of prices both above and below that average. Some models are marketed to more frugal shoppers and cost as little as $\$ 30.00$, while other backpacks are meant to appeal to high-end customers and cost $\$ 70.00$ or more.

Wearability: The construction of a backpack influences its comfort. Factors to consider include materials, stitching, straps, and padding. Packs can be made of canvas, nylon, polyester, rubber, or some combination of these. Stitches can be hidden or exposed; exposed stitches that make contact with the body can cause chaffing. Strap adjustment helps ensure proper fit, and padding ranges from $1 / 2$ inch to $1 \frac{1}{2}$ inches thick. A thicker padding keeps the body from being jabbed by the contents of the bag.

\section{Study 2: Vacation Destinations}

Weather: This refers to the typical climate you can expect at the two locations. The weather may be sunny and pleasant, rainy and overcast, or somewhere in between.

Beaches: This refers to the attractiveness of the beaches at the two locations. Beaches may be small, medium, or large. They may be clean and attractive, or dirty and ugly. 
Hotel: This describes the quality of the hotel you would be staying in. Hotels can be old and run-down, new and up-todate, or somewhere in between.

Water Temperature: This describes the warmth of the water along the beaches. Water temperature ranges from very cold to moderate to very warm.

Nightlife: This indicates the amount of nightlife in the nearby area. Some vacation areas have virtually no clubs, restaurants, etc. in the proximity. Other areas have a bit more nightlife, and some offer numerous possibilities.

\section{Study 3: Hotels}

Location: The hotel's location refers to where it is situated relative to other attractions. Some hotels are within walking distance to grocery stores, restaurants, and nightclubs, while others are farther away. Another important factor is the distance to the nearest beach. The beaches themselves can be public or private, and they vary in size from quite small to extremely large.

Pool: This refers to characteristics of the pool area operated and maintained by the hotel. Pools can vary across a range of sizes from small to enormous, and they may or may not have Jacuzzis or a sauna nearby. Hotel pools sometimes offer amenities such as swim-up bars or an outdoor grill/eatery. Some hotels also have an indoor pool.

Services: The hotels provide numerous services that can help make your stay more pleasant. Many hotels offer a morning meal-the breakfast hours end earlier at some hotels than at others, and the meals offered range from simple continental breakfasts to elaborate breakfast buffets. The television stations available on the in-room TV may include only local channels, or an extensive array of cable stations may be offered. Rooms may or may not have VCRs, and hotels may or may not rent movies at the front desk. Another factor to consider is the availability and cost of local telephone calls.

\section{References}

Ariely, D., J. Levav. 2000. Sequential choice in group settings: Taking the road less traveled and less enjoyed. J. Consumer Res. 27(December) 279-290.

Ariely, D., T. S. Wallsten. 1995. Seeking subjective dominance in multidimensional space: An explanation of the asymmetric dominance effect. Organ. Behavior Human Decision Processes 63(September) 223-232.

Bargh, J. A. 1996. Automaticity in social psychology. E. T. Higgins, A. W. Kruglanski, eds. Social Psychology: Handbook of Basic Principles. Guilford Press, New York.

Baron, J. 1997. Biases in the quantitative measurement of values for public decisions. Psych. Bull. 122(1) 72-88.

Bettman, J. R., M. Sujan. 1987. Effects of framing on evaluation of comparable and noncomparable alternatives by expert and novice consumers. J. Consumer Res. 14(September) 141-154.

Bettman, J. R., M. F. Luce, J. W. Payne. 1998. Constructive consumer choice processes. J. Consumer Res. 25(December) 187-217.

Bless, H., E. R. Igou, N. Schwartz, M. Waenke. 2000. Reducing context effects by adding context information: The direction and size of context effects in political judgment. Personality Soc. Psych. Bull. 26(September) 1036-1045.

Bond, S. D., K. A. Carlson, M. G. Meloy, J. E. Russo, R. S. Tanner. 2006. Precommitment bias in the evaluation of a single option. Working paper, Duke University, Durham, NC.
Carlson, K. A., L. K. Pearo. 2004. Limiting predecisional distortion by prior valuation of attribute components. Organ. Behavior Human Decision Processes 94(1) 48-59.

Carlson, K. A., J. Edward Russo. 2001. Biased interpretation of evidence by mock jurors. J. Experiment. Psych.: Appl. 7(June) 91-103.

Carlson, K. A., M. G. Meloy, J. E. Russo. 2006. Leader-driven primacy: Using attribute order to affect consumer choice. J. Consumer Res. Forthcoming.

Chaiken, S., S. Yates. 1985. Affective-cognitive consistency and thought-induced attitude polarization. J. Personality Soc. Psych. 49(6) 1470-1481.

Coupey, E., J. R. Irwin, J. W. Payne. 1998. Product category familiarity and preference construction. J. Consumer Res. 24(March) 459-468.

Delquie, P. 1993. Inconsistent trade-offs between attributes: New evidence in preference assessment biases. Management Sci. 39 1382-1395.

Delquie, P. 1997. Bimatching: A new preference assessment method to reduce compatibility effects. Management Sci. 43 640-658.

Delquie, P. 2003. Optimal conflict in preference assessment. Management Sci. 49 102-115.

Dhar, R., S. M. Nowlis, S. J. Sherman. 1999. Comparison effects on preference construction. $J$. Consumer Res. 26(December) 293-306.

Dhar, R., S. M. Nowlis, S. J. Sherman. 2000. Trying hard or hardly trying: An analysis of context effects in choice. J. Consumer Psych. 9(December) 189-200.

Duckworth, K. L., J. A. Bargh, M. Garcia, S. Chaiken. 2002. The automatic evaluation of novel stimuli. Pysch. Sci. 13(6) 513-519.

Einhorn, H. J. 1972. Expert measurement and mechanical combination. Organ. Behavior Human Performance 7(February) 86-106.

Ganzach, Y. 1995. Attribute scatter and decision outcome: Judgment versus choice. Organ. Behavior Human Decision Processes 62(April) 113-122

Garcia, M. T., J. A. Bargh. 2003. Automatic evaluation of novel words: The role of superficial phonetics. J. Language Soc. Psych. 22(December) 414-443.

Greenwald, A. G., S. C. Draine, R. L. Abrams. 1996. Three cognitive markers of unconscious semantic activation. Science 273(September) 1699-1702.

Gregory, R., S. Lichtenstein, P. Slovic. 1993. Valuing environmental resources: A constructive approach. J. Risk Uncertainty 7(March) 177-197.

Hammond, J. S., R. L. Keeney, H. Raiffa. 1999. Smart Choices: A Practical Guide to Making Better Decisions. Harvard Press, Cambridge, MA.

Higgins, E. T. 1996. Knowledge activation: Accessibility, applicability, and salience. E. T. Higgins, A. W. Kruglanski, eds. Social Psychology: Handbook of Basic Principles. Guilford Press, New York, 133-168.

Hsee, C. 1996. The evaluability hypothesis: An explanation for preference reversals between joint and separate evaluations of alternatives. Organ. Behavior Human Decision Processes 67(September) 247-257.

Hsee, C. K., Y. Rottenstreich. 2004. Music, pandas, and muggers: On the affective psychology of value. J. Experiment. Psych:: General 133(1) 23-30.

Huber, J., J. W. Payne, C. P. Puto. 1982. Adding asymmetrically dominated alternatives: Violations of regularity and the similarity hypothesis. J. Consumer Res. 9(June) 90-98.

Huber, J., D. R. Wittink, J. A. Fiedler, R. Miller. 1993. The effectiveness of alternative preference elicitation procedures in predicting choice. J. Marketing Res. 30(February) 105-114.

Janiszewski, C., M. Cunha. 2004. The influence of price discount framing on the evaluation of a bundle. J. Consumer Res. 30(March) 534-546. 
Janiszewski, C., T. Silk, A. D. J. Cooke. 2003. Different scales for different frames: The role of subjective scales and experience in explaining attribute framing effects. J. Consumer Res. 30(December) 311-325.

Jarvis, W. B. G., R. E. Petty. 1996. The need to evaluate. J. Personality Soc. Psych. 70(January) 172-194.

Johnson, E. J., J. W. Payne. 1985. Effort and accuracy in choice. Management Sci. 31(April) 394-414.

Keeney, R. L. 1992. Valued-Focused Thinking: A Path to Creative Decision Making. Harvard Press, Cambridge, MA.

Keeney, R. L. 1996. Valuing billions of dollars. R. J. Zechauser, R. L. Keeney, J. K. Sebenius, eds. Wise Choices: Decisions, Games, and Negotiations. Harvard Press, Cambridge, MA, 63-80.

Kivetz, R., I. Simonson. 2000. The effects of incomplete information on consumer choice. J. Marketing Res. 37(November) 427-448.

Kleinmuntz, D. N. 1990. Decomposition and the control of error in decision-analytic models. R. M. Hogarth, ed. Insights in Decision Making: A Tribute to Hillel J. Einhorn. University of Chicago Press, Chicago, IL, 107-126.

Lichtenstein, S., P. Slovic. 1971. Reversals of preference between bids and choices in gambling decisions. J. Experiment. Psych. 89(July) 46-55.

Livingston, R. W., M. B. Brewer. 2002. What are we really priming? Cue-based versus category-based processing of facial stimuli. J. Personality Soc. Psych. 82(1) 5-18.

Lynch, J. G. 1985. Uniqueness issues in the decompositional modeling of multiattribute overall evaluations: An information integration perspective. J. Marketing Res. 22(February) 1-19.

Mandel, N., E. J. Johnson. 2002. When web pages influence choice: Effects of visual primes on experts and novices. J. Consumer Res. 29(September) 235-245.

Meloy, M. G., J. E. Russo. 2004. Binary choice under instructions to select versus reject. Organ. Behavior Human Decision Processes 93(April) 114-128.

Montgomery, H. 1983. Decision rules and the search for a dominance structure: Towards a process model of decision making. P. C. Humphreys O. Svenson, A. Vari, eds. Analyzing and Aiding Decision Processes. North-Holland, Amsterdam, The Netherlands, 343-369.

Montgomery, H., H. Willen. 1999. Decision making and action: The search for a good structure. P. Juslin, H. Montgomery, eds. Judgment and Decision Making: Neo-Brunswickian and ProcessTracing Approaches. Lawrence Erlbaum Associates, Mahwah, NJ, 147-173.

Musch, J., K. C. Klauer. 2003. The Psychology of Evaluation: Affective Processes in Cognition and Emotion. Lawrence Erlbaum Associates, Mahwah, NJ.

Novemsky, N., R. Dhur, I. Simonson, N. Schwarz. 2003. Preference fluency and its effect on no-choice, compromise, and attraction effects. Presentation, Association for Consumer Research, Toronto, Ontario, Canada.

Nowlis, S. M., B. E. Kahn, R. Dhar. 2002. Coping with ambivalence: The effect of removing a "fence sitting" option on consumer attitude and preference judgments. J. Consumer Res. 29(December) 319-334.

Payne, J. W. 1982. Contingent decision behavior. Psych. Bull. 92(September) 382-402.

Payne, J. W., J. R. Bettman, E. Johnson. 1992. Behavioral decision research: A constructive processing perspective. Ann. Rev. Psych. 43(February) 87-131.

Payne, J. W., J. R. Bettman, D. A. Schkade. 1999. Measuring constructed preferences: Toward a building code. J. Risk Uncertainty 19(December) 243-270.
Plott, C. R. 1996. Rational individual behavior in markets and social choice processes. K. J. Arrow, E. Colombatto, M. Perlman, C. Schmidt, eds. The Rational Foundations of Economic Behavior. St. Martins Press, New York, 225-250.

Ratner, R. K., B. E. Kahn. 2002. The impact of private versus public consumption on variety-seeking behavior. J. Consumer Res. 29(September) 246-257.

Russo, J. E., K. A. Carlson. 2002. Individual decision-making. B. A. Weitz, R. Wensley, eds. Handbook of Marketing. Sage, Thousand Oaks, CA, 371-408.

Russo, J. E., P. J. H. Schoemaker. 2002. Winning Decisions: Getting It Right the First Time. Random House, New York.

Russo, J. E., V. H. Medvec, M. G. Meloy. 1996. The distortion of information during decisions. Organ. Behavior Human Decision Processes 66(April) 102-110.

Russo, J. E., M. G. Meloy, T. J. Wilks. 2000. Predecisional distortion of information by auditors and salespersons. Management Sci. 46(January) 13-27.

Schoemaker, J. H., J. C. Hershey. 1992. Utility measurement: Signal, noise, and bias. Organ. Behavior Human Decision Processes 52(January) 397-424.

Sen, S. 1998. Knowledge, information mode, and the attraction effect. J. Consumer Res. 25(June) 64-77.

Shafir, E. 1993. Choosing versus rejecting: Why some options are both better and worse than others. Organ. Behavior Human Decision Processes 55(July) 325-378.

Simonson, I. 1989. Choice based on reasons: The case of attraction and compromise effects. J. Consumer Res. 16(September) 158-174.

Simonson, I., A. Tversky. 1992. Choice in context: Tradeoff contrast and extremeness aversion. J. Marketing Res. 29(August) 281-295.

Slovic, P., 1995. The construction of preference. Amer. Psychologist 50(May) 364-371.

Slovic, P., B. Fischhoff, S. Lichtenstein. 1977. Behavioral decision theory. Ann. Rev. Psych. 28(February) 1-39.

Smith, J. E., D. von Winterfeldt. 2004. Decision analysis in management science. Management Sci. 50(May) 561-574.

Svenson, O. 1996. Decision making and the search for fundamental psychological regularities: What can be learned from a process perspective? Organ. Behavior Human Decision Processes 65(March) 252-267.

Tversky, A., D. Kahneman. 1986. Rational choice and the framing of decisions. J. Bus. 59(October) S251-S278.

Tversky, A., S. Sattath, P. Slovic. 1988. Contingent weighting in judgment and choice. Psych. Rev. 95(July) 371-384.

Tyszka, T., M. Wielochowski. 1991. Must boxing verdicts be biased? J. Behavioral Decision Making 4(December) 283-295.

von Winterfeldt, D., W. Edwards. 1986. Decision Analysis and Behavioral Research. Cambridge University Press, New York.

Wedell, D. H. 1997. Another look at reasons for choosing and rejecting. Memory Cognition 25(November) 873-887.

Wedell, D. H. 1998. Testing models of trade-off contrast in pairwise choice. J. Experiment. Psych.: Human Perception Performance 24(1) $49-65$.

Wright, P., M. A. Kriewall. 1980. State-of-mind effects on the accuracy with which utility functions predict marketplace choice. J. Marketing Res. 17(August) 277-293.

Zajonc, R. B. 1980. Feeling and thinking: Preferences need no inferences. Amer. Psychologist 35(February) 151-175.

Zajonc, R. B. 2001. Mere exposure: A gateway to the subliminal. Current Directions Psych. Sci. 10(December) 224-228. 
Copyright 2006, by INFORMS, all rights reserved. Copyright of Management Science is the property of INFORMS: Institute for Operations Research and its content may not be copied or emailed to multiple sites or posted to a listserv without the copyright holder's express written permission. However, users may print, download, or email articles for individual use. 